\title{
microRNAs and lipid metabolism
}

\author{
Binod Aryal ${ }^{1,2}$, Abhishek K. Singh ${ }^{1,2}$, Noemi Rotllan ${ }^{1}$, Nathan Price ${ }^{1}$, and Carlos \\ Fernández-Hernando ${ }^{1, *}$ \\ ${ }^{1}$ Vascular Biology and Therapeutics Program, Integrative Cell Signaling and Neurobiology of \\ Metabolism Program, Section of Comparative Medicine, and Department of Pathology, Yale \\ University School of Medicine, 10 Amistad St., New Haven, CT 06510. USA
}

\begin{abstract}
Purpose of review-Work over the last decade has identified the important role of microRNAs (miRNAS) in regulating lipoprotein metabolism and associated disorders including metabolic syndrome, obesity and atherosclerosis. This review summarizes the most recent findings in the field, highlighting the contribution of miRNAs in controlling low-density lipoprotein (LDL) and high-density lipoprotein (HDL) metabolism.
\end{abstract}

Recent findings-A number of miRNAs have emerged as important regulators of lipid metabolism, including miR-122 and miR-33. Work over the last two years has identified additional functions of miR-33 including the regulation of macrophage activation and mitochondrial metabolism. Moreover, it has recently been shown that miR-33 regulates vascular homeostasis and cardiac adaptation in response to pressure overload. In addition to miR-33 and miR-122, recent GWAS have identified single nucleotide polymorphisms (SNP) in the proximity of miRNAs genes associated with abnormal levels of circulating lipids in humans. Several of these miRNA, such as miR-148a and miR-128-1, target important proteins that regulate cellular cholesterol metabolism, including the low-density lipoprotein receptor (LDLR) and the ATP-binding cassette A1 (ABCA1).

Summary-microRNAs have emerged as critical regulators of cholesterol metabolism and promising therapeutic targets for treating cardiometabolic disorders including atherosclerosis. Here, we discuss the recent findings in the field highlighting the novel mechanisms by which miR-33 controls lipid metabolism and atherogenesis and the identification of novel miRNAs that regulate LDL metabolism. Finally, we summarize the recent findings that identified miR-33 as an important non-coding RNA that controls cardiovascular homeostasis independent of its role in regulating lipid metabolism.

\section{Keywords}

miRNAs; miR-33; miR-148a; atherosclerosis; cholesterol metabolism

\footnotetext{
*Corresponding author: Carlos Fernández-Hernando. Phone: +1 (203)-737-4615. carlos.fernandez@ yale.edu.

${ }^{2}$ These authors contributed equally to this work

CONFLIT OF INTEREST

There are not conflicts of interest.
} 


\section{INTRODUCTION}

The leading cause of death worldwide is cardiovascular disease (CVD). Atherosclerosis is the cause of the most common forms of CVD such as heart attack and stroke [1, 2]. Among others, the most important risk factors and mediators of atherosclerosis are the circulating levels of LDL-C and HDL-C $[1,2]$.

MicroRNAs (miRNAs) are 22-nt-long non-coding sequences involved in the posttranscriptional regulation of gene expression [3-5]. Since their initial discovery in Caenorhabditis elegans, these short non-coding RNAs have been involved in the regulation of a wide spectrum of biological processes, from development and metabolic regulation, to aging and disease progression [3-5]. miRNAs control the expression of numerous mRNA targets, and a single mRNA can be regulated by several miRNAs. Indeed, it has been proposed that the expression of more than half of the human genes are regulated by numerous miRNAs. miRNAs assemble with Argonaute proteins into miRNA-induce silencing complexes (miRISCs) to direct post-transcriptional silencing of complementary mRNA targets [3-5].

The role of miRNAs in the biology and pathophysiology of cardiovascular diseases (CVD) has been extensively studied over the past decades $[6,7]$. Of note, miRNAs have recently been uncovered as critical regulators of circulating lipids, a major factor that influences the risk of CVD. Increased levels of high-density lipoprotein (HDL) are associated with reduced risk for developing CVD. A number of miRNAs regulate different steps of HDL-C metabolism, from synthesis to clearance, of which miR-33a/b are the most well-studied [813]. While the role of miRNAs in regulating HDL-C metabolism has been deeply studied, the importance of miRNAs in controlling low-density lipoprotein (LDL) metabolism have fallen behind. Nonetheless, a number of recent reports have given light the importance of miRNAs in controlling plasma LDL-C levels, such as miR-148a, miR-128-1 or miR-30c that regulate genes involved in very low-density lipoprotein (VLDL) secretion, cholesterol biosynthesis and hepatic LDL receptor (LDLR) expression [14-17]. Thus, miRNAs have arisen as critical regulators of cholesterol metabolism and promising therapeutic targets for the treatment of CVD. Their conservation between species suggests that the biological pathways where miRNAs play a role may have been conserved. For that reason, miRNAs have a therapeutic potential, and different approaches have been undertaken to examine this possibility. In this review, we summarize the most important and novel roles of miRNAs in regulating lipoprotein metabolism.

\section{miRNA regulation of VLDL-C and LDL-C metabolism}

Increased levels of circulating ApoB-containing lipoproteins (VLDL and LDL) leads to the infiltration and retention of these lipoproteins in the arterial wall, which is a critical initiating event in the development of atherosclerosis [1,2]. Early studies uncovered the role of miR-122 in regulating LDL-C levels in mice and non-human primates [18, 19]. Antagonism of miR-122 in both animal models significantly reduces plasma LDL-C and VLDL-C levels $[18,19]$. These results were further confirmed in two independent miR-122 deficient mouse models [20, 21]. While the reduction in circulating ApoB-containing lipoproteins in miR-122 deficient mice and mice treated with miR-122 inhibitors is very well established, 
the molecular mechanisms and the mRNA targets that mediate this effect remain unknown. Further studies will be important to elucidate how hepatic miR-122 levels control lipoprotein metabolism. In addition to miR-122, recent reports have identified additional miRNAs that control plasma LDL-C levels. Using two independent approaches, human GWAS and a high-throughput genome-wide screening assay in vitro, the Näär and Fernández-Hernando laboratories identified miR-148a and miR-128-1 as important regulators of LDL-C and HDL-C metabolism $[14,17]$. Additional recent studies found miR-30c to be an important regulator of VLDL secretion through targeting of the microsomal transfer protein (MTP), which controls ApoB lipidation and hepatic VLDL production [15, 16]. Here, we summarize how these miRNAs control lipoprotein metabolism.

\section{miR-148a}

miR-148a is highly expressed in mouse and human hepatic tissue and conserved among vertebrate species. miR-eQTL analysis of human livers strongly support the fact that SNP status in the promoter region of miR-148a strongly correlated with altered levels of total cholesterol, LDL-C and triglycerides (TAGs) in circulation[14, 17, 22-24]. miR-148a directly targets the $3^{\prime} \mathrm{UTR}$ of $L D L R$, along with other genes involved in lipid metabolism, such as ATP-binding cassette A1 ( $A B C A 1)$, peroxisome proliferator-activated receptor gamma coactivator 1-alpha (PGC1a), AMP-activated protein kinase ( $A M P K)$ and Insulininduced gene 1 (INSIGI). Importantly, overexpression of miR-148a in mice reduces hepatic LDLR and ABCA1 expression, thus increasing circulating LDL-C and reducing plasma HDL-C levels [14, 17]. Conversely, antagonism of miR-148a in $A p o B^{T G} \mathrm{Ldlr}^{-/+}$and in $A \mathrm{poe}^{-/-}$mice decreases circulating LDL-C and increases plasma HDL-C [14, 17]. In addition to the liver, miR-148a is also expressed in a number of other tissues, including adipose tissue and hematopoietic cells. Interestingly, GWAS have associated SNPs in the miR-148a locus to obesity [25]. Moreover, miR-148a is highly expressed in macrophages and controls ABCA1 expression and cholesterol efflux [17]. Taken together, these findings suggest that miR-148a antisense oligonucleotide therapy could be useful for treating dyslipidemia and CVD. Further studies should test this possibility.

\section{mir-128-1}

In humans, miR-128 is encoded within an intron of the R3HDM1 gene on chromosome 2, and appears to be co-expressed with its host gene in numerous tissues [17]. Physiological studies have revealed that miR-128-1 plays a key role in regulating cholesterol/lipid and energy homeostasis. Longterm inhibition of miR-128-1 in hyperlipidemic $A p o e^{-/-}$mice leads to a marked decrease in circulating VLDL-C/LDL-C, VLDL-associated TAGs, and hepatic steatosis [17]. Similar to miR-148a, miR-128-1 also controls circulating lipoprotein metabolism by directly targeting the $3^{\prime} \mathrm{UTR}$ of $L D L R$ and $A B C A 1$ [17]. miR-128-1 antagonism also improves glucose clearance by enhancing hepatic insulin sensitivity. In addition to regulation of lipoprotein metabolism, miR-128-1 regulates ABCA-1 expression in macrophages, and its inhibition increases ABCA1 expression and macrophage cholesterol efflux. While these findings suggest that miR-148 and miR-128-1 antagonism might be promising therapeutic approaches for treating dyslipidemia, obesity and CVD, additional studies will be important to demonstrate efficacy in other animal models and examine the possible deleterious effects of long-term silencing. 
miR-30c

miR-30c has been identified in association with altered levels of circulating TC, TAGs and VLDL biogenesis [16]. miR-30c inhibits MTP, thus reducing VLDL-C production. Interestingly, miR-30c inhibits hepatic lipid synthesis by targeting lysophosphatidyl glycerol acyltransferase 1 (LPGAT1), which is involved in phospholipid synthesis. Importantly miR-30c overexpression using lentivirus in vivo markedly reduces circulating VLDL-C and LDL-C and improves atherosclerotic plaque burden in $\mathrm{Apoe}^{-/-}$mice. Similar results were obtained when miR-30c was overexpressed using miRNA mimics. As expected by its role in suppressing hepatic MTP expression, antagonism miR-30c increases plasma VLDL-C and LDL-C levels and promotes atherogenesis. Of note, miR-30c inhibits VLDL production without causing hepatic steatosis because also inhibits hepatic lipid synthesis, suggesting that miR-30c overexpression might be an attractive approach for treating subjects with homozygous hypercholesterolemia.

\section{microRNAs and HDL-C metabolism}

Since cholesterol cannot be degraded, mammalian cells orchestrate the removal of excess cholesterol from the peripheral tissues to liver for reutilization and secretion to feces through a process known as reverse cholesterol transport (RCT). As the first step of RCT, nascent HDL particles are generated in the liver and intestine through the efflux of cholesterol and phospholipids to lipid poor apolipoprotein A-1 (ApoA1) particles. ABCA1 plays a key role in HDL formation as evidenced by a near-absence of plasma HDL in patients with Tangier disease, who have mutations in the $A B C A 1$ gene [26]. In addition to ABCA1, ABCG1 also facilitates cholesterol efflux to HDL particles, whereas scavenger receptor class B type 1 (SR-BI) contributes to HDL maturation and mediates the delivery of cholesterol to the liver and other steroidogenic tissues [27, 28]. Within the liver, part of the cholesterol is enzymatically converted into bile acid salts in a multistep process initiated by enzyme cholesterol 7a-hydroxylase (CYP7A1) [29]. As a final step of RCT, these highly soluble biliary lipids are secreted from the hepatocytes with the help of three different transmembrane transporters: ATP-binding cassette, sub-family B, member 11 (ABCB11), ATP-binding cassette, sub-family G, member 5/8 (ABCG5/ABCG8) and ATP-binding cassette, sub-family B, member 4 (ABCB4) [30]. In addition, another transporter, aminophospholipid transporter, class I, type 8B, member 1 (ATP8B1) maintains the asymmetry of phospholipids required for proper membrane function [31]. Over the past decade, miRNAs have emerged as important regulators of HDL metabolism and RCT, including direct targeting of genes involved in cellular cholesterol efflux, HDL biogenesis, hepatic HDL uptake, and bile acid synthesis and secretion [32]. In addition to their role in regulating HDL metabolism, HDL-enriched miRNAs regulate gene expression in recipient cells, thus providing an exciting novel mechanism that could explain a part of the antiatherogenic effect of HDL [33, 34]. Our group and others have identified numerous miRNAs including miR-33a/b, miR-144, miR-148a and miR-128 that are important regulators of HDL biology, particularly in the context of atherosclerosis [11-13, 35-38]. 


\section{miR-33 and HDL-C metabolism}

The sterol response element-binding proteins are master regulators of sterol and fatty acid synthesis [39]. miR-33 family of microRNAs consists of $m i R-33 a$ and $m i R-33 b$, which are encoded within the introns of the SREBP2 and SREBP1 genes respectively, and are coexpressed in different stimulatory conditions $[12,13,32]$. Both miR-33 isoforms share the same seed sequence but differ in 2 nucleotides in the $3^{\prime}$ region. The relevance of miR-33 on sterol metabolism was initially revealed in studies showing that miR-33 modulated the expression of $A B C A 1$ and $A B C G 1$ both in mouse hepatocytes and macrophages. Overexpression of miR-33 in mouse liver resulted in a decrease in $A B C A 1$ and $A B C G 1$ expression and a parallel decrease in the plasma HDL-C levels. Alternatively, the antagonism of miR-33 expression using antisense oligonucleotides (ASO-33) resulted in an increase in hepatic $A B C A 1 / A B C G 1$ expression and plasma HDL-C levels [11-13]. Genetic ablation of $m i R-33$ recapitulated the results from ASO studies, as the $m i R-33^{-/}$mice had increased liver $A B C A 1$ expression and elevated plasma HDL-C indicating the physiological relevance of miR-33 in regulation of HDL-C metabolism [10]. Most of these studies were performed through therapeutic and genetic modulation of miR-33 in mice [40]. However, rodents have only one isoform of miR-33 (equivalent of miR-33a in humans), but not miR-33b. Although miR-33a and miR-33b have same seed sequence and same predicted targets, studies have been done in non-human primates to address the role of both isoforms in HDL-C metabolism. Treatment of African green monkeys with antisense inhibitors against miR-33 resulted in an increase in plasma HDL-C levels [37, 41]. In addition, Horie et al recently developed miR-33b knockin mice in an intron of Srebp1 gene [42].

Macrophages from these mice had reduced $A B C A 1$ and $A B C G 1$ expression and cholesterol efflux capacity to ApoA1 and HDL. In addition, knockin heterozygous mice had a 35\% reduction in HDL-C levels compared to wild-type counterparts confirming the physiological role of miR-33b in regulating HDL-C metabolism [42].

Remarkably, miR-33 has also been reported to influence cholesterol efflux independent of its ability to regulate $A B C A 1 / A B C G 1$ expression. Karunakaran et al have demonstrated that inhibition of miR-33 increases mitochondrial respiration and ATP production through upregulation of miR-33 target genes including peroxisome proliferator-activated receptor $\gamma$ coactivator 1-a (PGC1-a), pyruvate dehydrogenase kinase isozyme 4 (PDK4), and solute carrier family 25 (SLC25A25), and promotes ABCA1- mediated cholesterol efflux [43]. In addition to regulating cholesterol efflux and HDL biogenesis, several studies have reported a role for miR-33 in regulating bile acid synthesis and secretion. Allen et al have shown that miR-33 regulates the expression of bile acid transporters including $A B C B 11$ and $A T P 8 B 1$, and as such systemic silencing of miR-33 leads to increased sterols in bile and enhanced RCT in vivo [44]. Taken together, these studies suggest an important role for both miR-33 family members in regulating HDL-C metabolism and RCT and atherosclerosis progression. Additionally, miR-33 might also modulate the inflammatory status of atherosclerotic plaques independent its regulation of HDL. It was shown that miR-33 inhibitors efficiently transduce macrophages accumulated in the subendothelial space of aortic root of $\mathrm{LdIr}^{-/-}$mice and promote their polarization towards anti-inflammatory M2 phenotype by direct targeting of $A M P K$. Another target of miR-33 is $A L D H 1 A 2$, a gene involved in retinoic acid 
metabolism, that is also derepressed in these macrophages resulting in the induction of regulatory $\mathrm{T}$ cells and atheroprotection [45].

In addition to regulating lipid accumulation through RCT, Ouimet et al has recently reported that miR-33 also promotes lipid accumulation in macrophages infected by Mycobacterium tuberculosis (Mtb) by suppressing lysosomal degradation and autophagy [46]. In particular, overexpression of miR-33 in Mtb infected macrophages repressed autophagy of Mtb, promoted lipid body formation, and repressed fatty acid oxidation by targeting genes that encode proteins involved in autophagosome formation (autophagy protein 5 (ATG5), ATG7, $A T G 12$, microtubule associated protein 1 light chain 3 beta $(M A P 1 L C 3 B)$, lysosomal function (lysosomal-associated membrane protein 1 ( $L A M P I)$, lysosomal acid lipase (LIPA) and the regulation of autophagy ( $U V R A G, A M P K$ ). Conversely, inhibition of miR-33 promoted the targeting and killing of Mtb by autophagy machinery [46]. In another recent study, Fessier and colleagues found that miR-33 regulates innate immune response via ABCA1 remodeling of membrane micro-domains. miR-33 augments macrophage lipid raft content and enhances pro-inflammatory cytokine induction and NFKB activation by LPS [47]. In contrast to Ouimet's study, the authors found that LPS markedly reduces the expression of miR-33, which correlates with a significant downregulation of Srebp2, the host gene for miR-33 [47]. In agreement with this observation, numerous studies have shown that SREBP2 expression is significantly reduced in macrophages stimulated with LPS [48, 49].

In addition the role of miR-33 in regulating lipid accumulation in macrophages and hepatocytes, Nishiga et al reported that miR-33 preserves lipid raft cholesterol content in cardiac fibroblasts through the regulation of $A B C A 1$ expression and maintains adaptive fibrotic response in the remodeling heart [50]. Surprisingly, miR-33 deficient mice showed impaired systolic function after thoracic aortic constriction (TAC). Interestingly, another recent study has demonstrated that miR-33 protects against neointimal hyperplasia induced by arterial mechanical stretch in grafted veins [51]. Mechanistically, the authors found that miR-33 targets bone morphogenetic protein 3 (BMP3) and attenuates vascular smooth muscle cell (VSMC) proliferation and neointimal expansion. These findings correlate with a number of studies that have linked miR-33 expression and cellular proliferation. Together, these findings suggest that miR-33 might regulate other cellular functions besides lipid metabolism.

A number of recent studies have shown that chronic antagonism or genetic ablation of miR-33 can cause adverse effects including dyslipidemia, obesity, hepatic steatosis and insulin resistance [52-54]. Horie and colleagues found that miiR-33 regulates the expression of SREBP1 and its absence increases hepatic SREBP1 levels and activation [54]. While the authors suggest that the increase levels of SREBP1 contributes to the marked obesity, dyslipidemia and insulin resistance observed in miR-33-deficient mice, early work for Brown and Goldstein's laboratory found a modest effect on body weight and circulating lipids in hepatic SREBP1a and SREBP1c transgenic mice $[55,56]$. These observations suggest that the obesity phenotype observed in the miR-33 deficient mice is likely mediated by its effect in several metabolic tissues and its regulation of other genes besides SREBP1. Further studies using tissue specific miR-33 knockout mice will help to dissect the specific 
contribution of miR-33 in several metabolic tissues how this contributes to the marked obesity and insulin resistance phenotype observed in the global miR-33 deficient mice.

In addition to miR-33, a number of other miRNAs have been reported to regulate different aspects of HDL-C metabolism including cellular cholesterol efflux, HDL biogenesis, and HDL uptake. Some of these miRNAs including miR-758, miR-144, miR-26, miR-27a/b, miR302a, miR-148a, miR-128-1, and miR-19b have been shown to regulate ABCA1 expression in macrophages and hepatocytes [14, 17, 57-62]. A recent study has shown that inhibition of miR-302a in macrophages results in increased $A B C A 1$ expression and subsequent cholesterol efflux to ApoA1 particles in vitro, while long term in vivo antagonism of miR-302a led to an enhanced liver ABCA1 expression and plasma HDL levels, and decreased atherosclerosis [61]. Similarly, it has been shown that miR-128-1 and miR-148a also contribute to the post-transcriptional regulation of ABCA1. Overexpression of miR-128-1 and miR-148a in mice significantly lowered circulating HDL-C and hepatic ABCA1 levels $[14,17]$. Most importantly, antagonism of miR-148a in vivo markedly increased hepatic ABCA1 expression and plasma HDL-C levels [14]. Interestingly, not all miRNAs that regulate $A B C A 1$ expression and cholesterol efflux in vitro influence plasma HDL-C levels. For instance, miR-27a/b modulates cholesterol efflux from hepatocytes and macrophages through the regulation of $A B C A 1$. However, in vivo manipulation of miR-27b did not influence plasma HDL-C levels in chow or high fat diet conditions possibly because of miR-27 targeting other lipid related genes angiopoietin like 3 (ANGPTL3) and glycerol-3-phosphate acyltransferase (GPAM) that counteract the influence on HDL-C levels through ABCA1 regulation [63, 64]. HDL transports cholesterol from peripheral tissues where it is selectively taken up by SR-BI receptors, a critical step of RCT. A number of miRNAs regulate the expression of SR-BI and thereby affect the uptake of HDL by the liver. In particular, overexpression of miR-455, miR-125a, miR-185, miR-96 and miR-223 attenuate SR-BI expression and HDL uptake in liver cell lines [65, 66]. Importantly, genetic ablation of miR-223 in mice enhances hepatic SR-BI and plasma HDL levels [67]. Due to space limitations, specific roles of other miRNAs are not discussed in this review. Altogether progress made in this field has demonstrated that a complex network of miRNAs regulate different stages of HDL metabolism by coordinating posttranscriptional control of multiple genes, and thus play an important role in the development of cardiovascular disorders (Figure).

\section{CONCLUDING REMARKS}

Over recent years, contributions of many researchers groups has made evident the important role of miRNAs in regulation of lipid metabolism and cardiovascular disease. In this review article, we have highlighted some of the most important and recent findings related to miRNA mediated regulation of HDL-C, LDL-C, and the development of atherosclerosis. These findings indicate that miRNA targeted therapies may serve as a novel approach for the treatment of CVD. However chronic treatment or genetic ablation of some of these miRNAs (miR-122 and miR-33) has been found to result in adverse effects including dyslipidemia, obesity, hepatic steatosis, and hepatocellular carcinoma. These findings along with an inability to demonstrate the specific mechanisms by which miRNAs exert these phenotypic effects has raised concerns about the use of miRNA based therapies. As such, future 
experiments will be important for elucidating the specific functions of miRNAs in different tissues and the contribution of individual miRNA targets toward mediating specific phenotypes. These steps will be necessary to facilitate the progress and assess the outcome of miRNA based clinical trials for CVDs.

\title{
Acknowledgments
}

\author{
None. \\ FINANCIAL SUPPORT AND SPONSORSHIP
}

C.F.-H. is supported in part by NIH Grant R35HL135820 and Foundation Leducq Transatlantic Network of Excellence in Cardiovascular Research and AHA Established Investigator Award (16EIA27550004).

\section{References}

Papers of particular interest, published within the annual period of review, have been highlighted as:

- of special interest

•• of outstanding interest

1. Glass CK, Witztum JL. Atherosclerosis. the road ahead. Cell. 2001; 104:503-516. [PubMed: 11239408]

2. Lusis AJ. Atherosclerosis. Nature. 2000; 407:233-241. [PubMed: 11001066]

3. Ambros V. The functions of animal microRNAs. Nature. 2004; 431:350-355. [PubMed: 15372042]

4. Bartel DP. MicroRNAs: target recognition and regulatory functions. Cell. 2009; 136:215-233. [PubMed: 19167326]

5. Filipowicz W, Bhattacharyya SN, Sonenberg N. Mechanisms of post-transcriptional regulation by microRNAs: are the answers in sight? Nature reviews. Genetics. 2008; 9:102-114. [PubMed: 18197166]

6. Fernandez-Hernando C, Ramirez CM, Goedeke L, Suarez Y. MicroRNAs in metabolic disease. Arterioscler Thromb Vasc Biol. 2013; 33:178-185. [PubMed: 23325474]

7. Olson EN. MicroRNAs as therapeutic targets and biomarkers of cardiovascular disease. Sci Transl Med. 2014; 6:239ps233.

8. Canfran-Duque A, Ramirez CM, Goedeke L, et al. microRNAs and HDL life cycle. Cardiovasc Res. 2014; 103:414-422. [PubMed: 24895349]

9. Fernandez-Hernando C, Suarez Y, Rayner KJ, Moore KJ. MicroRNAs in lipid metabolism. Curr Opin Lipidol. 2011; 22:86-92. [PubMed: 21178770]

10. Horie T, Ono K, Horiguchi M, et al. MicroRNA-33 encoded by an intron of sterol regulatory element-binding protein 2 (Srebp2) regulates HDL in vivo. Proc Natl Acad Sci U S A. 2010; 107:17321-17326. [PubMed: 20855588]

11. Marquart TJ, Allen RM, Ory DS, Baldan A. miR-33 links SREBP-2 induction to repression of sterol transporters. Proc Natl Acad Sci U S A. 2010; 107:12228-12232. [PubMed: 20566875]

12. Najafi-Shoushtari SH, Kristo F, Li Y, et al. MicroRNA-33 and the SREBP host genes cooperate to control cholesterol homeostasis. Science. 2010; 328:1566-1569. [PubMed: 20466882]

13. Rayner KJ, Suarez Y, Davalos A, et al. MiR-33 contributes to the regulation of cholesterol homeostasis. Science. 2010; 328:1570-1573. [PubMed: 20466885]

14••. Goedeke L, Rotllan N, Canfran-Duque A, et al. MicroRNA-148a regulates LDL receptor and ABCA1 expression to control circulating lipoprotein levels. Nat Med. 2015; 21:1280-1289. This study identify microRNAs that regulates LDLr activity using a genome-wide screen approach. The authors found that overexpressing miR-148a attenuates the expression of LDLr and ABCA1 in human hepatic cell lines. Most importatly, the authors demonstarte that antagonism miR-148a 
in mice markadly increases hepatic LDLr and ABCA1, decrease circulating LDL-C and increase plasma HDL-C levels. [PubMed: 26437365]

15•. Irani S, Pan X, Peck BC, et al. MicroRNA-30c Mimic Mitigates Hypercholesterolemia and Atherosclerosis in Mice. J Biol Chem. 2016; 291:18397-18409. This study demonstrates that overexpressing miR-30c mimics attenuates VLDL production and protect agains atherosclerosis in Apoe $^{-/-}$mice. [PubMed: 27365390]

16. Soh J, Iqbal J, Queiroz J, et al. MicroRNA-30c reduces hyperlipidemia and atherosclerosis in mice by decreasing lipid synthesis and lipoprotein secretion. Nat Med. 2013; 19:892-900. [PubMed: 23749231]

17••. Wagschal A, Najafi-Shoushtari SH, Wang L, et al. Genome-wide identification of microRNAs regulating cholesterol and triglyceride homeostasis. Nat Med. 2015; 21:1290-1297. This GWAS identify microRNAs associated with plasma lipid levels in humans. Importantly, the authors found that genetic variations encoded within the miR-148a and miR-128a genomic loci influence plasma LDL-C and TAGs levels. [PubMed: 26501192]

18. Elmen J, Lindow M, Schutz S, et al. LNA-mediated microRNA silencing in non-human primates. Nature. 2008; 452:896-899. [PubMed: 18368051]

19. Esau C, Davis S, Murray SF, et al. miR-122 regulation of lipid metabolism revealed by in vivo antisense targeting. Cell Metab. 2006; 3:87-98. [PubMed: 16459310]

20. Castoldi M, Vujic Spasic M, Altamura S, et al. The liver-specific microRNA miR-122 controls systemic iron homeostasis in mice. J Clin Invest. 2011; 121:1386-1396. [PubMed: 21364282]

21. Hsu SH, Wang B, Kota J, et al. Essential metabolic, anti-inflammatory, and anti-tumorigenic functions of miR-122 in liver. J Clin Invest. 2012; 122:2871-2883. [PubMed: 22820288]

22. Do R, Willer CJ, Schmidt EM, et al. Common variants associated with plasma triglycerides and risk for coronary artery disease. Nat Genet. 2013; 45:1345-1352. [PubMed: 24097064]

23. Global Lipids Genetics C. Willer CJ, Schmidt EM, et al. Discovery and refinement of loci associated with lipid levels. Nat Genet. 2013; 45:1274-1283. [PubMed: 24097068]

24. Huan T, Rong J, Liu C, et al. Genome-wide identification of microRNA expression quantitative trait loci. Nature communications. 2015; 6:6601.

25. Shi C, Zhang M, Tong M, et al. miR-148a is Associated with Obesity and Modulates Adipocyte Differentiation of Mesenchymal Stem Cells through Wnt Signaling. Sci Rep. 2015; 5:9930. [PubMed: 26001136]

26. Bodzioch M, Orso E, Klucken J, et al. The gene encoding ATP-binding cassette transporter 1 is mutated in Tangier disease. Nat Genet. 1999; 22:347-351. [PubMed: 10431237]

27. Kennedy MA, Barrera GC, Nakamura K, et al. ABCG1 has a critical role in mediating cholesterol efflux to HDL and preventing cellular lipid accumulation. Cell metabolism. 2005; 1:121-131. [PubMed: 16054053]

28. Valacchi G, Sticozzi C, Lim Y, Pecorelli A. Scavenger receptor class B type I: a multifunctional receptor. Annals of the New York Academy of Sciences. 2011; 1229:E1-7. [PubMed: 22239457]

29. Chiang JY. Bile acids: regulation of synthesis. Journal of lipid research. 2009; 50:1955-1966. [PubMed: 19346330]

30. Esteller A. Physiology of bile secretion. World J Gastroenterol. 2008; 14:5641-5649. [PubMed: 18837079]

31. Paulusma CC, Folmer DE, Ho-Mok KS, et al. ATP8B1 requires an accessory protein for endoplasmic reticulum exit and plasma membrane lipid flippase activity. Hepatology. 2008; 47:268-278. [PubMed: 17948906]

32. Rottiers V, Naar AM. MicroRNAs in metabolism and metabolic disorders. Nature reviews Molecular cell biology. 2012; 13:239-250. [PubMed: 22436747]

33. Tabet F, Vickers KC, Cuesta Torres LF, et al. HDL-transferred microRNA-223 regulates ICAM-1 expression in endothelial cells. Nat Commun. 2014; 5:3292. [PubMed: 24576947]

34. Vickers KC, Palmisano BT, Shoucri BM, et al. MicroRNAs are transported in plasma and delivered to recipient cells by high-density lipoproteins. Nat Cell Biol. 2011; 13:423-433. [PubMed: 21423178]

35. Gelissen IC, Harris M, Rye KA, et al. ABCA1 and ABCG1 synergize to mediate cholesterol export to apoA-I. Arterioscler Thromb Vasc Biol. 2006; 26:534-540. [PubMed: 16357317] 
36. Ramirez CM, Rotllan N, Vlassov AV, et al. Control of Cholesterol Metabolism and Plasma HDL Levels by miRNA-144. Circ Res. 2013

37. Rayner KJ, Esau CC, Hussain FN, et al. Inhibition of miR-33a/b in non-human primates raises plasma HDL and lowers VLDL triglycerides. Nature. 2011; 478:404-407. [PubMed: 22012398]

38. Rayner KJ, Sheedy FJ, Esau CC, et al. Antagonism of miR-33 in mice promotes reverse cholesterol transport and regression of atherosclerosis. J Clin Invest. 2011; 121:2921-2931. [PubMed: 21646721]

39. Horton JD, Goldstein JL, Brown MS. SREBPs: activators of the complete program of cholesterol and fatty acid synthesis in the liver. J Clin Invest. 2002; 109:1125-1131. [PubMed: 11994399]

40. Rotllan N, Ramirez CM, Aryal B, et al. Therapeutic silencing of microRNA-33 inhibits the progression of atherosclerosis in Ldlr-/- mice-brief report. Arterioscler Thromb Vasc Biol. 2013; 33:1973-1977. [PubMed: 23702658]

41. Rottiers V, Obad S, Petri A, et al. Pharmacological inhibition of a microRNA family in nonhuman primates by a seed-targeting 8-mer antimiR. Sci Transl Med. 2013; 5:212ra162.

42. Horie T, Nishino T, Baba O, et al. MicroRNA-33b knock-in mice for an intron of sterol regulatory element-binding factor 1 (Srebf1) exhibit reduced HDL-C in vivo. Sci Rep. 2014; 4:5312. [PubMed: 24931346]

43•. Karunakaran D, Thrush AB, Nguyen MA, et al. Macrophage Mitochondrial Energy Status Regulates Cholesterol Efflux and Is Enhanced by Anti-miR33 in Atherosclerosis. Circulation research. 2015; 117:266-278. This study describes the role of miR-33 in regulating mitochondrial energy status, influencing macrophage cholesterol efflux. [PubMed: 26002865]

44. Allen RM, Marquart TJ, Albert CJ, et al. miR-33 controls the expression of biliary transporters, and mediates statin- and diet-induced hepatotoxicity. EMBO Mol Med. 2012; 4:882-895. [PubMed: 22767443]

45•. Ouimet M, Ediriweera HN, Gundra UM, et al. MicroRNA-33-dependent regulation of macrophage metabolism directs immune cell polarization in atherosclerosis. The Journal of clinical investigation. 2015; 125:4334-4348. This study describes additional molecular mechanisms by which inhibition of miR-33 influences macrophage polarization towars M2-like phenotype and attenuates atherosclerosis in mice. [PubMed: 26517695]

46. Ouimet M, Koster S, Sakowski E, et al. Mycobacterium tuberculosis induces the miR-33 locus to reprogram autophagy and host lipid metabolism. Nat Immunol. 2016; 17:677-686. [PubMed: 27089382]

47. Lai L, Azzam KM, Lin WC, et al. MicroRNA-33 Regulates the Innate Immune Response via ATP Binding Cassette Transporter-mediated Remodeling of Membrane Microdomains. J Biol Chem. 2016; 291:19651-19660. [PubMed: 27471270]

48. Dennis EA, Deems RA, Harkewicz R, et al. A mouse macrophage lipidome. J Biol Chem. 2010; 285:39976-39985. [PubMed: 20923771]

49. Reboldi A, Dang EV, McDonald JG, et al. Inflammation. 25-Hydroxycholesterol suppresses interleukin-1-driven inflammation downstream of type I interferon. Science. 2014; 345:679-684. [PubMed: 25104388]

50• Nishiga M, Horie T, Kuwabara Y, et al. MicroRNA-33 Controls Adaptive Fibrotic Response in the Remodeling Heart by Preserving Lipid Raft Cholesterol. Circulation research. 2016 This study describes a novel role of miR-33 in regulating lipid ratfs content in cardiofibroblast and cardiac fibrosis in response to pressure overload.

51 • Huang K, Bao H, Yan ZQ, et al. MicroRNA-33 protects against neointimal hyperplasia induced by arterial mechanical stretch in the grafted vein. Cardiovasc Res. 2017 This study describes a novel role of miR-33 in regulating VSMC proliferation and neointimal expanssion in grafted veins.

52. Allen RM, Marquart TJ, Jesse JJ, Baldan A. Control of very low-density lipoprotein secretion by N-ethylmaleimide-sensitive factor and miR-33. Circ Res. 2014; 115:10-22. [PubMed: 24753547]

53. Goedeke L, Salerno A, Ramirez CM, et al. Long-term therapeutic silencing of miR-33 increases circulating triglyceride levels and hepatic lipid accumulation in mice. EMBO Mol Med. 2014; 6:1133-1141. [PubMed: 25038053]

54. Horie T, Nishino T, Baba O, et al. MicroRNA-33 regulates sterol regulatory element-binding protein 1 expression in mice. Nat Commun. 2013; 4:2883. [PubMed: 24300912] 
55. Shimano H, Horton JD, Hammer RE, et al. Overproduction of cholesterol and fatty acids causes massive liver enlargement in transgenic mice expressing truncated SREBP-1a. J Clin Invest. 1996; 98:1575-1584. [PubMed: 8833906]

56. Shimano H, Horton JD, Shimomura I, et al. Isoform 1c of sterol regulatory element binding protein is less active than isoform 1a in livers of transgenic mice and in cultured cells. J Clin Invest. 1997; 99:846-854. [PubMed: 9062341]

57. Ramirez CM, Davalos A, Goedeke L, et al. MicroRNA-758 regulates cholesterol efflux through posttranscriptional repression of ATP-binding cassette transporter A1. Arterioscler Thromb Vasc Biol. 2011; 31:2707-2714. [PubMed: 21885853]

58. Ramirez CM, Rotllan N, Vlassov AV, et al. Control of cholesterol metabolism and plasma highdensity lipoprotein levels by microRNA-144. Circulation research. 2013; 112:1592-1601. [PubMed: 23519695]

59. Sun D, Zhang J, Xie J, et al. MiR-26 controls LXR-dependent cholesterol efflux by targeting ABCA1 and ARL7. FEBS Lett. 2012; 586:1472-1479. [PubMed: 22673513]

60. Zhang M, Wu JF, Chen WJ, et al. MicroRNA-27a/b regulates cellular cholesterol efflux, influx and esterification/hydrolysis in THP-1 macrophages. Atherosclerosis. 2014; 234:54-64. [PubMed: 24608080]

61. Meiler S, Baumer Y, Toulmin E, et al. MicroRNA 302a is a novel modulator of cholesterol homeostasis and atherosclerosis. Arteriosclerosis, thrombosis, and vascular biology. 2015; 35:323331.

62. Lv YC, Tang YY, Peng J, et al. MicroRNA-19b promotes macrophage cholesterol accumulation and aortic atherosclerosis by targeting ATP-binding cassette transporter A1. Atherosclerosis. 2014; 236:215-226. [PubMed: 25084135]

63. Goedeke L, Rotllan N, Ramirez CM, et al. miR-27b inhibits LDLR and ABCA1 expression but does not influence plasma and hepatic lipid levels in mice. Atherosclerosis. 2015; 243:499-509. [PubMed: 26520906]

64. Vickers KC, Shoucri BM, Levin MG, et al. MicroRNA-27b is a regulatory hub in lipid metabolism and is altered in dyslipidemia. Hepatology. 2013; 57:533-542. [PubMed: 22777896]

65. Hu Z, Shen WJ, Kraemer FB, Azhar S. MicroRNAs 125a and 455 repress lipoprotein-supported steroidogenesis by targeting scavenger receptor class B type I in steroidogenic cells. Mol Cell Biol. 2012; 32:5035-5045. [PubMed: 23045399]

66. Wang L, Jia XJ, Jiang HJ, et al. MicroRNAs 185, 96, and 223 repress selective high-density lipoprotein cholesterol uptake through posttranscriptional inhibition. Mol Cell Biol. 2013; 33:1956-1964. [PubMed: 23459944]

67. Vickers KC, Landstreet SR, Levin MG, et al. MicroRNA-223 coordinates cholesterol homeostasis. Proc Natl Acad Sci U S A. 2014; 111:14518-14523. [PubMed: 25246565] 


\section{KEY POINTS}

1. miRNAs are key regulators of lipid metabolism and genetic variations in miRNA loci have been associated with alterations in circulating cholesterol and triglycerides (TAG) in humans.

2. miR-33 controls HDL-C metabolism and other cellular functions associated with cardiovascular disease, including macrophage activation, mitochondrial biogenesis, and autophagy.

3. Further studies are necessary to determine whether silencing of miR-148a and miR-128-1 will attenuate atherosclerosis, obesity and insulin resistance. 


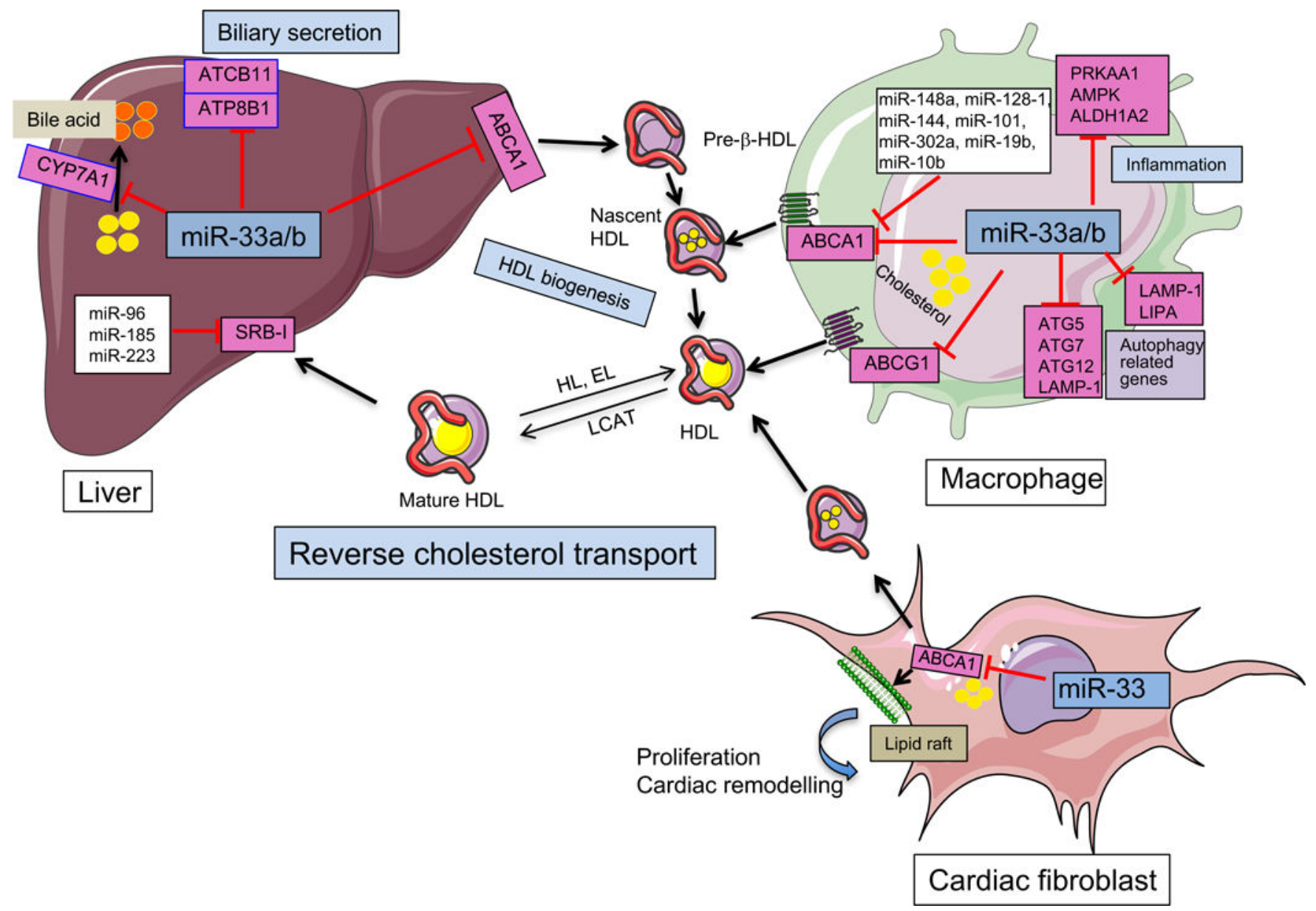

Figure. miRNA regulation of HDL-C metabolism

ABCA1, a major transporter that regulates HDL biogenesis and cholesterol efflux in macrophages accumulated in the artery wall, is regulated by a number of miRNAs including miR-33. miR-33 controls numerous steps of the reverse cholesterol transport pathway by regulating the expression of numerous genes associated with HDL biogenesis (ABCA1), cholesterol efflux in peripheral tissues including macrophages and cardiac fibroblasts [ABCA1 and ABCG1 (only in rodents)] and bile acid synthesis (CYP7A1) and secretion (ABCB11 and ATP8B1)in liver. In addition, miR-33 also promotes lipid accumulation in macrophages and favors Mtb survival by targeting the expression of key autophagy effectors ATG5, ATG7, ATG12, LAMP-1 and LIPA and controls macrophage polarization by regulating the expression of PRKAA1, AMPK and ALDH1A2. In addition to miR-33, ABCA1 is highly regulated at the post-transcriptional level in several tissues by numerous miRNAs including miR-148a, miR-144, miR-101, miR-128, miR-27a/b, miR-302a and miR-10b. Free cholesterol in nascent HDL is further esterified to cholesteryl esters by lecithin-cholesterol acyltransferase (LCAT) leading to the formation of mature HDL particles. HDL particles deliver cholesterol to the liver via the SRB1 receptor, which is also regulated by several miRNAs including miR-185, miR-223, and miR-96. This figure was performed using the Servier Medical Art illustration resources. 